\title{
BMJ Open Determinants of mortality and prolonged hospital stay among dengue patients attending tertiary care hospital: a cross-sectional retrospective analysis
}

\author{
Tauqeer Hussain Mallhi, ${ }^{1}$ Amer Hayat Khan, ${ }^{1}$ Azmi Sarriff, ${ }^{1}$ Azreen Syazril Adnan, ${ }^{2}$ \\ Yusra Habib Khan ${ }^{1}$
}

To cite: Mallhi TH, Khan AH, Sarriff A, et al. Determinants of mortality and prolonged hospital stay among dengue patients attending tertiary care hospital: a cross-sectional retrospective analysis. BMJ Open

2017;7:e016805. doi:10.1136/ bmjopen-2017-016805

- Prepublication history for this paper is available online. To view these files please visit the journal online (http://dx.doi org/10.1136/bmjopen-2017016805).

Received 14 March 2017 Revised 9 May 2017 Accepted 25 May 2017

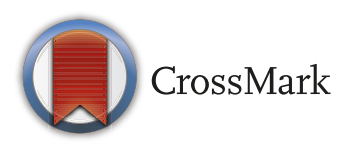

${ }^{1}$ Discipline of Clinical Pharmacy, School of Pharmaceutical Sciences, Universiti Sains Malaysia, Penang, Malaysia ${ }^{2}$ Chronic Kidney Disease Resource Centre, School of Medical Sciences, Health Campus, Universiti Sains Malaysia, Kelantan, Malaysia

\section{Correspondence to} Dr Tauqeer Hussain Mallhi; tauqeer.hussain.mallhi@otmail. com and Amer Hayat Khan; dramer2006@gmail.com

\section{ABSTRACT}

Objectives Dengue imposes substantial economic, societal and personal burden in terms of hospital stay, morbidity and mortality. Early identification of dengue cases with high propensity of increased hospital stay and death could be of value in isolating patients in need of early interventions. The current study was aimed to determine the significant factors associated with denguerelated prolonged hospitalisation and death.

Design Cross-sectional retrospective study.

Setting Tertiary care teaching hospital.

Participants Patients with confirmed dengue diagnosis were stratified into two categories on the basis of prolonged hospitalisation ( $\leq 3$ days and $>3$ days) and mortality (fatal cases and non-fatal cases). Clinicolaboratory characteristics between these categories were compared by using appropriate statistical methods. Results of 667 patients enrolled, 328 (49.2\%) had prolonged hospitalisation. The mean hospital stay was $4.88 \pm 2.74$ days. Multivariate analysis showed that dengue haemorrhagic fever (OR 2.3), elevated alkaline phosphatase (ALP) (OR 2.3), prolonged prothrombin time (PT) (OR 1.7), activated partial thromboplastin time (aPTT) (OR 1.9) and multiple-organ dysfunctions (OR 2.1) were independently associated with prolonged hospitalisation. Overall case fatality rate was $1.1 \%$. Factors associated with dengue mortality were age $>40$ years $(p=0.004)$, secondary infection $(p=0.040)$, comorbidities $(p<0.05)$, acute kidney injury $(p<0.001)$, prolonged PT $(p=0.022)$, multiple-organ dysfunctions $(p<0.001)$, haematocrit $>20 \%$ $(p=0.001)$, rhabdomyolosis $(p<0.001)$ and respiratory failure $(p=0.007)$. Approximately half of the fatal cases in our study had prolonged hospital stay of greater than three days.

Conclusions The results underscore the high proportion of dengue patients with prolonged hospital stay. Early identification of factors relating to prolonged hospitalisation and death will have obvious advantages in terms of appropriate decisions about treatment and management in high dependency units.

\section{INTRODUCTION}

Dengue is among the most important arthropod-borne disease that has rapidly been spreading in several regions of the world
Strengths and limitations of this study

To the best of the authors' knowledge, this is the first study to evaluate predisposing factors of prolonged hospitalisation among dengue patients in Malaysia.

- This study involved heterogeneous group of patients from tertiary-level teaching hospital of Malaysia, a tropical country with hyperendemic nature of dengue, and hence its findings can be generalised to other tropical regions.

- We analysed commonly available clinico-laboratory features of patients with dengue as predisposing factors that enhance the clinical applicability of current study in medical practice.

- Early recognition of factors identified in current study can potentially improve patients' outcomes, which in turn can translate to reduced denguerelated hospital stay and mortality.

- The admission and discharge criteria may vary among various clinicians attending patients and may interfere with the results of our findings. However, in our institution patients are routinely discharged on the basis of laboratory findings and clinical conditions.

in recent years. The disease is widespread throughout the tropics, with local variations in risk, influence by rainfall, temperature and unplanned rapid urbanisation. ${ }^{1}$ The spectrum of disease varies from mild self-limiting illness, dengue fever (DF) to more severe and fulminating forms, dengue haemorrhagic fever (DHF) and dengue shock syndrome (DSS). ${ }^{2}$ World Health Orgaization (WHO) estimates that about $40 \%$ of the world's population live in areas having risk of dengue transmission. The surge in dengue has been most marked in Asia, with an estimated 1.8 billion people at risk of dengue. ${ }^{3}$ Southeast Asia in particular has seen large epidemics of the disease in recent years with attendant mortality from dengue viral infection (DVI). ${ }^{4}$ 


\begin{tabular}{rrr}
\hline & Cases & Deaths \\
\hline $\mathbf{1 9 9 5}$ & 6534 & $\mathbf{2 8}$ \\
$\mathbf{1 9 9 6}$ & 14255 & 32 \\
$\mathbf{1 9 9 7}$ & 19429 & $\mathbf{5 2}$ \\
$\mathbf{1 9 9 8}$ & 27381 & 82 \\
$\mathbf{1 9 9 9}$ & 101146 & 37 \\
\hline $\mathbf{2 0 0 0}$ & 7103 & 45 \\
$\mathbf{2 0 0 1}$ & 16368 & 50 \\
$\mathbf{2 0 0 2}$ & 32767 & 99 \\
$\mathbf{2 0 0 3}$ & 31545 & $\mathbf{7 2}$ \\
$\mathbf{2 0 0 4}$ & 33895 & 102 \\
$\mathbf{2 0 0 5}$ & 39654 & 107 \\
\hline $\mathbf{2 0 0 6}$ & 38556 & 92 \\
$\mathbf{2 0 0 7}$ & 48846 & 98 \\
$\mathbf{2 0 0 8}$ & 49335 & 112 \\
$\mathbf{2 0 0 9}$ & 41486 & 88 \\
\hline $\mathbf{2 0 1 0}$ & 46171 & 134 \\
\hline $\mathbf{2 0 1 1}$ & 19884 & 36 \\
\hline $\mathbf{2 0 1 2}$ & 21900 & 35 \\
$\mathbf{2 0 1 3}$ & 43346 & 92 \\
\hline $\mathbf{2 0 1 4}$ & 108698 & 215 \\
\hline $\mathbf{2 0 1 5}$ & 120836 & 336 \\
\hline
\end{tabular}
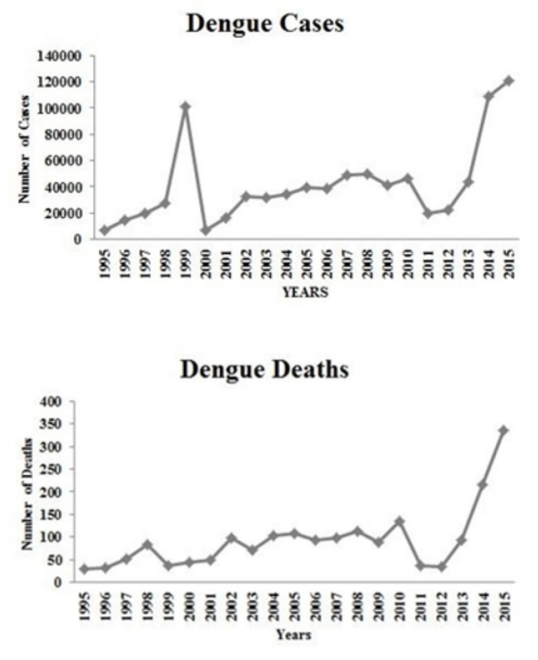

Figure 1 Incidence of dengue cases and deaths in Malaysia (Ministry of Health Malaysia, epidemiological data of WHO and $\mathrm{MOH}$ may slightly vary due to the differences in reporting criteria or lack of reporting).

Currently, Malaysia is the leading country in terms of the number of dengue cases reported worldwide. ${ }^{5}$ Dengue is among top five notifiable diseases in the country $^{6}$ and continues to be a formidable public health concern. Malaysia is hyperendemic for dengue and is experiencing worst dengue crisis nowadays, while some countries in Southeast Asia, including the Philippines and Thailand, have seen decreases in DVI activity in 2014. Reported dengue cases in Malay Archipelago in 2014 increased dramatically (160\%) to 98128 with 189 deaths compared with 37698 with 79 deaths in the previous year. ${ }^{7-9}$ It is worth mentioning that the year 2015 painted a scary picture as the highest number of dengue cases $(\mathrm{n}=111285)$, along with 301 deaths, ever recorded in the history of the country (figure 1) ${ }^{10}$ More recently, 94 dengue-related deaths have been reported to WHO until April 2016 compared with a total of 120 deaths during the same reporting period in 2015. ${ }^{3}$

The case fatality rate (CFR) due to dengue varies among countries, but can be as high as $10 \%-15 \%$ in some and $<1 \%$ in others. ${ }^{11} 12$ The recent epidemiology of dengue in Malaysia has imposed substantial economic and disease burden on patients and healthcare system with increased hospital stay, high morbidity and attendant mortality. Despite exponential increase in dengue-related deaths in Malaysia, data describing clinico-laboratory characteristics and factors associated with fatal cases are scarce. Efforts made by Sam et al lack statistical comparison between fatal and non-fatal cases and are limited to DHF cases. ${ }^{13}$ These findings necessitate the urgent need of studies to differentiate clinico-laboratory characteristics among fatal and non-fatal dengue cases. On the other hand, we previously reported that DVI requires longer hospital stay irrespective of the disease severity, resulting in significant burden in terms of health service costs. This is of particular importance in resource-limited setting, especially in dengue endemic regions. ${ }^{14}$ Early identification of risk factors associated with prolonged hospital stay and mortality can help physicians to primacies the management of high-risk patients with dengue. These factors could also be used in formulating predictive score to identify severely ill patients during future outbreaks and to prioritise care that may translate into reduced morbidity and mortality. In this context, a retrospective case series was intended to investigate clinico-laboratory characteristics associated to the prolonged hospitalisation ( $>3$ days) and mortality among patients with dengue admitted to the tertiary care hospital.

\section{MATERIALS AND METHODS Data anonymisation}

All data were analysed anonymously and, hence, informed consent was not required. The patients were identified from a central computerised record with their registration number $(\mathrm{RN})$. Data of the cases were retrieved and specific numeral codes were given to each case before data analysis. Identity of all patients was not disclosed in current study.

\section{Study location and population}

The current study was conducted in HUSM, tertiary-level teaching hospital with 950 beds that serves an estimated 1.4-1.8 million inhabitants of Kelantan. Kelantan is an agrarian state located in the northeast of Peninsular Malaysia and is among the top five dengue hotspots in the country where the dengue cases are substantially rising every year. Malays are the major (95\%) ethnic group in Kelantan while Chinese constitute merely $4 \%$ of state population. The hospital also serves as a referral centre for nearby states to treat severe and complicated dengue cases and has reliable medical records. ${ }^{145}$

All the patients with dengue admitted to the hospital during the period of 6years (January 2008 to December 2013) were included into the study. Patients aged $\geq 12$ years admitted with primary and confirmed diagnosis of DVI, irrespective of severity, were identified by RN using hospital record management system. The process of patients' selection and identification, along with inclusion and exclusion criteria, is described in figure 2.

\section{Diagnosis of dengue}

Suspected dengue cases were confirmed by laboratory criteria that were further subjected to clinical case definition of DVI. Suspected dengue infection was defined as the presence of fever and any two of the following symptoms: myalgia, headache, arthralgia, skin rash, retro-orbital pain, haemorrhagic manifestation(s) or leucopenia (white blood cell (WBC) count of $<4 \times 10^{9} \mathrm{~L}^{-1}$ ). Suspected cases were confirmed by using at least one of the following criteria: (i) positive reverse transcriptase PCR result, (ii) presence of dengue immunoglobulin $\mathrm{M}$ and $\mathrm{G}$ antibodies in acute-phase serum by ELISA (Pan Bio Dengue IgM ELISA, Dengue IgM 
Ethical Approval from JEPEM-USM

(USM/JEPeM/14080278)

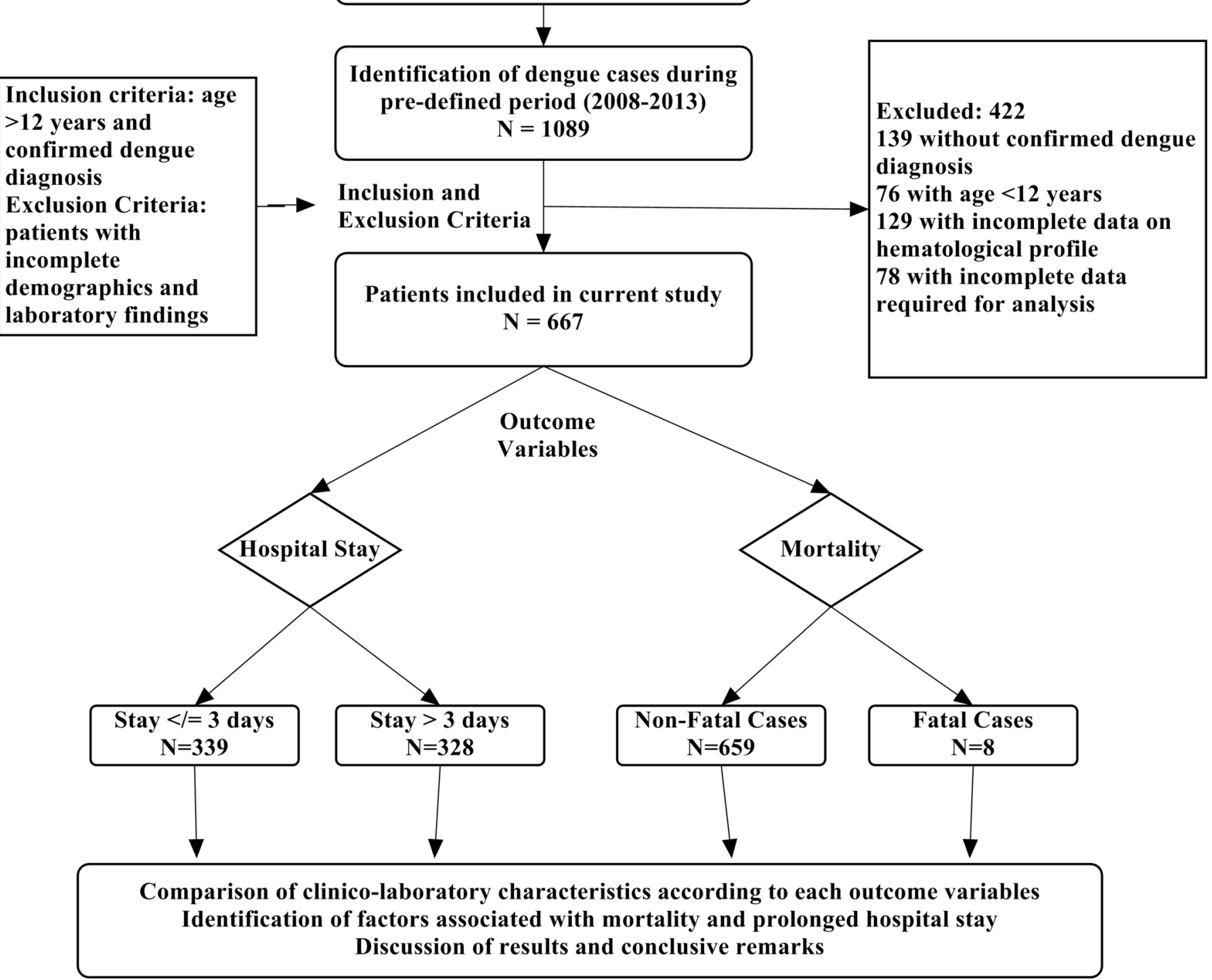

Figure 2 Methodological flow of study. JEPeM, Human Resource Ethics Committee.

Dot Enzyme Immunoassay, SD Dengue IgM and IgG capture ELISA Kits; Standard Diagnostics, Korea) and (iii) at least fourfold increase of dengue-specific haemagglutination inhibition titres in convalescent serum compared with acute-phase serum. The serum samples were also tested for dengue-specific NS1 (pan-E Early dengue ELISA kit by Panbio, Australia, and Platelia dengue NS1Ag assay by Bio-Rad Laboratories, USA). Only confirmed dengue cases were included in analysis. Primary dengue infection was distinguished from secondary infection by using IgM-IgG ratio where dengue infection was defined as primary if ratio $\geq 1.8$ and as secondary if $<1.8$ or if there was a fourfold increase of HAI and the titres were $\leq 1: 1280$ and $\geq 1: 2560$, respectively. ${ }^{14}$ Serologically confirmed patients with dengue were subjected to clinical case definition and disease severity was classified into DF, DHF and DSS, according to the WHO criteria. ${ }^{16}$

\section{Data collection and management}

All the required data were collected on structured data collection form, approved by hospital ethical committee. After identification of patients with confirmed dengue, numeral codes were given to patients and these codes were used as identifier during data analysis. Usually patients with dengue infection have hospital stay between 3 and 4 days; ${ }^{14}{ }^{17}$ therefore, we used $>3$ days as cut-off point for prolonged hospitalisation (median hospital stay in the present study was 3 days). Patients having hospital stay $\leq 3$ days were compared with those staying $>3$ days in order to identify possible predictors of increased hospitalisation. Similarly, we stratified all patients into fatal and 
non-fatal cases and their clinical and laboratory characteristics were compared. Patients' demographics and clinical presentations were recorded on day of admission while laboratory findings were recorded for each day of hospitalisation until discharge or death, whichever occurred first.

\section{Definitions}

For the purpose of current study, terms used were defined as follows.

Hospital stay is defined by $\geq 1$ day bed occupancy in hospital; mortality means death within 14 days after admission; hypokalaemia $(\mathrm{K}<3.5 \mathrm{mmol} / \mathrm{L})$; hyponatremia $(\mathrm{Na}<135 \mathrm{mmol} / \mathrm{L}$ ); oliguria (UO $<400 \mathrm{~mL} /$ day after 24 hours of appropriate hydration); hypotension (blood pressure $<110 / 70 / 70 \mathrm{~mm} \mathrm{Hg}$ ) ; elevated transaminases (elevation of liver enzymes such as aspartate aminotransferase $($ AST) and/or alanine aminotransferase (ALT) $>2$ times the normal value); prolonged prothrombin time $(\mathrm{PT}>15 \mathrm{~s})$; prolonged activated partial thromboplastin time ( $\mathrm{aPTT}>35 \mathrm{~s}$ ); urinary sedimentations (presence of glycosuria, haematuria, proteinuria, leucocytouria, urine pus, urine epithelial cells); anaemia ( $\mathrm{Hb}<12 \mathrm{~g} / \mathrm{dL})$; DVI (DF, DHF, DSS); acute kidney injury (AKI) (Acute Kidney Injury Network (AKIN) of classification); stages of AKI based on serum creatinine values (AKIN-I, AKINII, AKIN-III); severe dengue (DHF, DSS); multiple-organ dysfunction (MOD) (dysfunction of $\geq 2$ organs); hepatic dysfunction (elevation of liver enzymes); and thrombocytopenia (platelets count $<100 \times 10^{9}$ cells). Reference values of laboratory parameters in the current study are according to the hospital pathology lab-included aspartate aminotransferase (AST) [5-34 IU/L]; alanine aminotransferase (ALT) [10-35 IU/L]; alkaline phos-

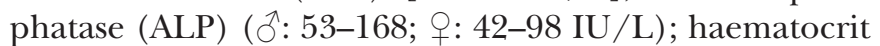
(ठ: 37.5-49.8; ㅇ: 31.8-42.4); platelets $\left(158-410 \times 10^{9} / \mathrm{L}\right.$ ); WBCs (ô: 3.8-9.7; 우: 3.4-10.1); PT (12-13s) and aPTT $(30-50 \mathrm{~s})$.

\section{Statistical analysis}

All the patients were divided into two groups based on presence or absence of outcomes (mortality, prolonged hospital stay). For quantitative variables, measures of central tendency and dispersion were calculated. Qualitative variables are presented as frequencies and proportions for which frequency served as the numerator and total number of patients $(n=667)$ served as the denominator. Relevant denominator was stated before proportion, where it varied. Comparison of categorical variables between two groups was done by using $\chi^{2}$ test (if at least $80 \%$ of cells have expected frequencies of five or more) or Fisher's exact test (if $<80 \%$ of cells have expected frequencies of five or more). Comparison of continuous variables was done by an independent Student's t-test. Logistic regression was used to estimate the associations between prolonged hospital stay, as the response variables, and potential predictors. These potential predictor variables were chosen on the basis of statistical significance and their biological plausibility with the outcomes. Co-linearity diagnostics was performed on variables selected for regression analysis. The strength of association was evaluated using an OR and a 95\% CI. The variables with univariate $p$ value $<0.25$ were subjected to multivariate analysis. ${ }^{18}$ The use of univariate $p$ values $<0.25$ has advantage of tending to include more variables in multivariate analysis while traditional levels of $\mathrm{p}$ value such as 0.05 can fail in identifying variables known to be important. ${ }^{19}$ Calibration of final multivariate logistic model (model fit) was assessed by Hosmer-Lameshow test. The two-sided statistical significance level, $p$ value, was set at 0.05 for all inferential analyses in this study. Data were compiled and analysed using Statistical Package for Social Sciences program V.20.

\section{RESULTS}

Out of the total dengue cases admitted to the hospital, 667 patients with mean age $30.8 \pm 16.1$ years were included in analysis (figure 2). According to WHO criteria, DF was diagnosed in $627(88.1 \%)$ patients while DHF (DHF grades I and II) and DSS (DHF grades III and IV) were observed in $74(11.1 \%)$ and $5(0.8 \%)$ cases, respectively. Most of the studied participants were ethnic Malay (90.4\%), followed by Chinese (7.9\%) and Indians (1.4). The most common non-haemorrhagic manifestations at hospital admission were fever (97\%), headache (58.9\%), retro-orbital pain $(25 \%)$, myalgia $(70.4 \%)$, arthralgia $(56.5 \%)$, nausea $(31.2 \%)$, vomiting $(54.2 \%)$, abdominal pain $(44.7 \%)$, cough $(18.5 \%)$, chills $(31.5 \%)$, anorexia $(27 \%)$, malaise $(7.3 \%)$, lethargy $(27.4 \%)$, palpitation (2\%), flushing $(19 \%)$, dehydration $(11 \%)$, ascites $(2.7 \%)$ and pleural effusion $(3.2 \%)$. On the other hand, petechia $(11.2 \%)$, haematemesis $(1.9 \%)$, purpura ecchymosis $(7.4 \%)$, epistaxis $(4.9 \%)$, haematuria $(2.5 \%)$ and gum bleeding $(9.4 \%)$ were common haemorrhagic presentations among patients with dengue.

The mean length of hospital stay was $4.88 \pm 2.74$ days (median 3, IQR 3, range 1-35 days). Prolonged hospitalisation ( $>3$ days) was seen in $49.2 \% \quad(n=328 / 7667)$ patients while length of stay (LOS) was $\leq 3$ days among $50.8 \%(\mathrm{n}=339 / 667)$ studied participants. Clinical characteristics of patients with and without prolonged LOS were compared (table 1). Patients having DHF, hypertension (HTN), elevated alkaline phosphatase (ALP), multiple organ dysfunctions (MODs), prolonged PT and aPTT were significantly associated with prolonged hospitalisation. Although other factors such as secondary infection, comorbidities, acute kidney injury (AKI), elevated aspartate aminotransferase (AST) and haematocrit $>20 \%$ were more profound among patients with prolonged hospitalisation, but their statistical association was insignificant $(p>0.05)$. It was interesting to note that male gender and patients with DF were more likely to stay $\leq 3$ days in the current study (table 1).

To determine the factors independently associated with prolonged hospitalisation, we developed a series of 
Table 1 Comparison of clinical characteristics (on-admission) of dengue patients according to the presence or absence of prolonged hospital stay (>3 days)

\begin{tabular}{|c|c|c|c|c|}
\hline \multirow[b]{2}{*}{ Variables } & \multirow{2}{*}{$\begin{array}{l}\text { Total cohort } \\
(n=667)\end{array}$} & \multicolumn{2}{|c|}{ LOS in hospital } & \multirow[b]{2}{*}{ p Value } \\
\hline & & $\leq 3$ days $(n=339)$ & $>3$ days $(n=328)$ & \\
\hline Age (years), mean $\pm S D$ & $30.8 \pm 16.1$ & $30.7 \pm 15.8$ & $30.7 \pm 16.5$ & 0.305 \\
\hline Age>40years, $n(\%)$ & $167(25 \%)$ & $84(24.5 \%)$ & $83(25.3 \%)$ & 0.875 \\
\hline Age>60years, $n(\%)$ & $35(5.2 \%)$ & $17(5.1 \%)$ & $18(5.5 \%)$ & 0.784 \\
\hline Male gender, n (\%) & $378(56.7 \%)$ & $189(55.8 \%)$ & $139(42.4 \%)$ & 0.626 \\
\hline Secondary infection, n (\%) & $73(10.9 \%)$ & $33(9.7 \%)$ & $40(12.2 \%)$ & 0.309 \\
\hline \multicolumn{5}{|l|}{ Dengue severity, n (\%) } \\
\hline DF, n (\%) & $588(88 \%)$ & $313(92.3 \%)$ & $275(83.8 \%)$ & 0.001 \\
\hline $\mathrm{DHF}^{\star}, \mathrm{n}(\%)$ & 79 (10.5\%) & $26(7.7 \%)$ & $53(16.2 \%)$ & 0.001 \\
\hline Warning signs, n (\%) & $271(40.6 \%)$ & $141(41.6 \%)$ & $130(39.6 \%)$ & 0.607 \\
\hline \multicolumn{5}{|l|}{ Comorbidities, n (\%) } \\
\hline $\mathrm{DM}, \mathrm{n}(\%)$ & $36(5.4 \%)$ & $14(4.1 \%)$ & $22(6.7 \%)$ & 0.141 \\
\hline HTN, n (\%) & $35(5.2 \%)$ & $12(3.5 \%)$ & $23(7 \%)$ & 0.044 \\
\hline CKD, n (\%) & $33(4.9 \%)$ & $15(4.4 \%)$ & $18(5.5 \%)$ & 0.522 \\
\hline IHD, n (\%) & $25(3.7 \%)$ & $9(2.7 \%)$ & $16(4.9 \%)$ & 0.131 \\
\hline Comorbidities $\geq 2, \mathrm{n}(\%)$ & $36(5.4 \%)$ & $13(3.8 \%)$ & $23(7 \%)$ & 0.069 \\
\hline AKI, n (\%) & $95(14.2 \%)$ & $43(12.7 \%)$ & $52(15.9 \%)$ & 0.242 \\
\hline Scr $\geq 2$ mg/dL, n (\%) & $29(4.1 \%)$ & $10(2.9 \%)$ & $19(5.8 \%)$ & 0.048 \\
\hline Elevated ALT, n (\%) & $362(54.3 \%)$ & $192(56.6 \%)$ & $170(51.8 \%)$ & 0.197 \\
\hline Elevated AST, n (\%) & $447(67 \%)$ & $226(66.7 \%)$ & $221(67.4 \%)$ & 0.636 \\
\hline Elevated ALP, n (\%) & $133(19.9 \%)$ & $44(13 \%)$ & $89(27.1 \%)$ & $<0.001$ \\
\hline Prolonged PT, n (\%) & $224(33.3 \%)$ & $80(23.6 \%)$ & $144(43.9 \%)$ & $<0.001$ \\
\hline Prolonged aPTT, n (\%) & $159(23.8 \%)$ & $52(15.3 \%)$ & 107 (32.6\%) & $<0.001$ \\
\hline Thrombocytopenia, n (\%) & $395(59.2 \%)$ & $191(56.3 \%)$ & $204(62.2 \%)$ & 0.134 \\
\hline MODs, n (\%) & $117(17.5 \%)$ & 44 (13\%) & $72(22.3 \%)$ & 0.002 \\
\hline Haematocrit>20\%, n (\%) & $73(10.9 \%)$ & $38(11.2 \%)$ & $35(10.7 \%)$ & 0.824 \\
\hline
\end{tabular}

$p$ values were calculated between patients with and without prolonged hospital stay.

${ }^{*} \mathrm{DHF}$ includes all four grades.

AKI, acute kidney injury; ALP, alkaline phosphatase; ALT, alanine aminotransferase; aPTT, activated partial thromboplastin time;

AST, aspartate aminotransferase; CKD, chronic kidney disease; DF, dengue fever; DHF, dengue haemorrhagic fever; DM, diabetes mellitus;

HTN, hypertension; IHD, ischaemic heart disease; LOS, length of stay; MODs, multiple organ dysfunction; PT, prothrombin time; Scr, serum creatinine.

logistic regression analyses, which are shown in table 2. Out of eight readily available clinical parameters that had $p$ values $<0.25$ in the univariate analysis, four factors (DHF, elevated ALP, increased PT/aPTT, MODs) were found to be independently associated with prolonged LOS. Although the presence of HTN and thrombocytopenia was found as risk factors of prolonged LOS in unadjusted analysis but neither of these showed statistically significant results in multivariate model. Though thrombocytopenia and AKI were insignificant variables in table 1 but included in logistic regression due to their hypothetical and clinical association with prolonged hospitalisation. Receiver-operating characteristics (ROC) curve analysis with area under the curve (AUC) as 0.812 demonstrated that logistic model has good ability to predict prolonged hospitalisation among patients with dengue(figure 3).

\section{Evaluation of dengue-related fatal cases}

The overall dengue CFR was $1.1 \%$ and all eight fatal cases were attributed to the dengue infection. Of the dengue deaths, four $(50 \%)$ were male, six $(75 \%)$ were Malay and two (25\%) were Chinese. The mean age was $48.8 \pm 25.6$ years (range 13-78) and most of the fatal cases $(\mathrm{n}=6 / 8,75 \%)$ were rural residents. DF was present in $6 / 8(75 \%)$ patients while DHF was recorded in $2 / 8$ $(25 \%)$ cases. Four patients $(50 \%)$ had no pre-existing comorbidities and the remaining four patients $(50 \%)$ were having at least two comorbid conditions (table 3 ).

The mean duration of hospital stay among death cases was 5.6 \pm 3.2 days (median 5, IQR 9, range 3-12 days) and all of them were admitted $\geq 5$ th day of onset of illness. The number of fatal cases in the present study was very small that precluded us to perform logistic regression analysis. 
Table 2 Univariate and multivariate analysis to evaluate determinants (risk factors) of prolonged length of hospital stay

\begin{tabular}{|c|c|c|c|c|c|c|}
\hline \multirow[b]{2}{*}{ Variables* $^{*}$} & \multicolumn{3}{|c|}{ Univariate analysis } & \multicolumn{3}{|c|}{ Multivariate analysis } \\
\hline & $p$ Value & OR & $95 \% \mathrm{Cl}$ & p Value & OR & $95 \% \mathrm{Cl}$ \\
\hline DHF & 0.001 & 2.3 & 1.4 to 3.8 & 0.009 & 2.3 & 1.2 to 4.3 \\
\hline Hypertension & 0.048 & 2.1 & 1.1 to 4.2 & 0.688 & 1.2 & 0.5 to 2.1 \\
\hline Elevated ALP & $<0.001$ & 2.5 & 1.7 to 3.7 & $<0.001$ & 2.3 & 1.5 to 3.5 \\
\hline Prolonged PT & $<0.001$ & 2.6 & 1.9 to 3.6 & 0.009 & 1.7 & 1.1 to 2.5 \\
\hline Prolonged aPTT & $<0.001$ & 2.7 & 1.8 to 3.9 & 0.005 & 1.9 & 1.2 to 2.9 \\
\hline Thrombocytopenia & 0.135 & 1.3 & 0.9 to 1.7 & 0.197 & 1.3 & 0.9 to 1.8 \\
\hline AKI & 0.242 & 1.3 & 0.8 to 2.0 & 0.174 & 0.7 & 0.3 to 1.2 \\
\hline MODs & 0.002 & 1.9 & 1.3 to 2.9 & 0.013 & 2.1 & 1.2 to 3.7 \\
\hline
\end{tabular}

Hosmer-Lameshow test statistics: $\chi^{2}: 7.474$, degree of freedom: 6 , p value $=0.279$.

*Variables lack multicollinearity and had variance inflation value $<2$.

AKI, acute kidney injury; ALP, alkaline phosphatase; aPTT, activated partial thromboplastin time; DHF, dengue haemorrhagic fever; MODs, multiple-organ dysfunction; PT, prothrombin time.

However, results of $\chi^{2}$ analysis demonstrated significantly higher proportion of age $>40$ years, secondary infection, comorbidities (diabetes mellitus (DM), ischaemic heart disease (IHD)), AKI, prolonged PT, MODs, haemoconcentration, rhabdomyolosis and respiratory failure among death cases as compared with patients who survived (table 3 ).

The main presenting complaints in all fatal cases are shown in table 4. Fever, nausea, vomiting and abdominal pain were presented in all death cases while retro-orbital pain and dysuria were present in 6/8 (75\%) patients.

Table 5 shows individual data points of all eight patients who died. All of them were brought alive to the hospital and succumbed to the infection within 3-12 days of

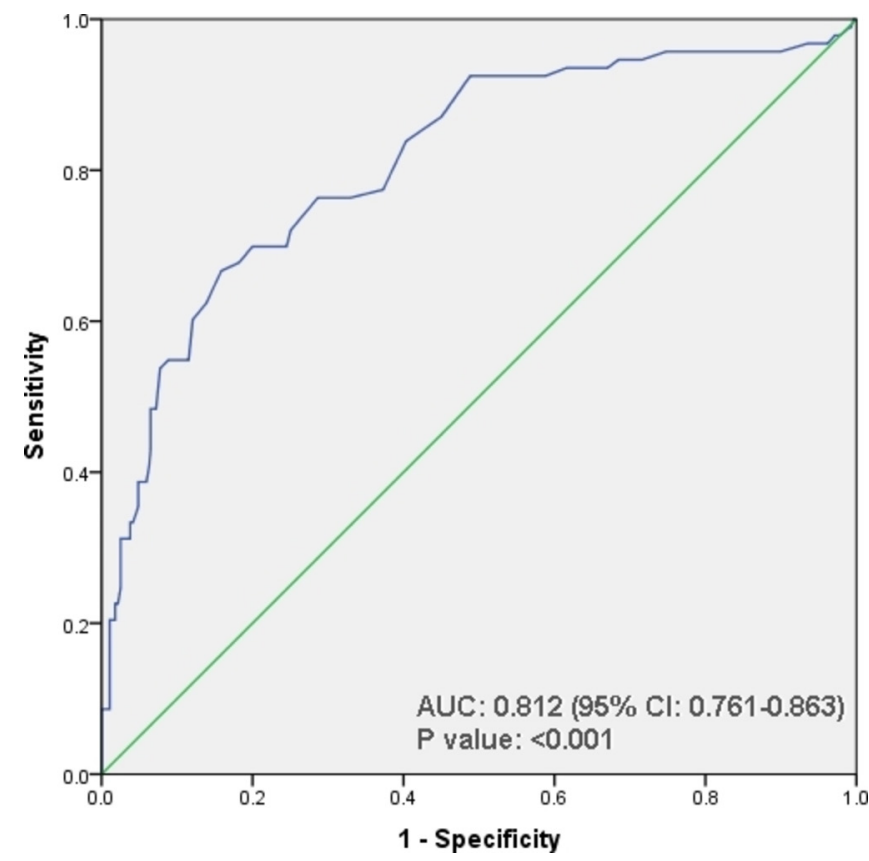

Figure 3 Receiver-operating characteristics curve analysis of multivariate logistic model predicting prolonged hospitalisation among dengue patients. AUC, area under the curve. admission. These patients were admitted on or after day 5 of illness and had rapid deterioration of their clinical features, resulting in admission to the intensive care unit. Dengue infection was confirmed in all patients by dengue serological tests. Three patients had clinical history relating to DF. About one-third patients died within 72 hours of hospital admission. On admission, all the cases were febrile (mean $38.9^{\circ} \mathrm{C}$ ) and about $50 \%$ patients had pulse rate $>100$ beats per minute. We observed multifactorial causes of death in the current study including dengue infection complicated with MODs (100\%), AKI $(100 \%)$, shock (25\%), acute respiratory distress syndrome $(25 \%)$, disseminated intravascular coagulation $(12.5 \%)$, gastric bleeds $(25 \%)$ and underlined comorbid conditions $(50 \%)$. Five dead patients $(62.5 \%)$ had prolonged hospital stay in the current study. Unfortunately, we had no access to postmortem and autopsy data of fatal cases.

\section{DISCUSSION}

The alarming rise of dengue epidemiology has been highlighted to haunt $40 \%$ of world's population. In Malaysia, dengue is perceived as a highly contagious health threat with escalating trend of infection. The average number of dengue cases and death tolls had recorded a high surge over the past few years, leading to substantial disease burden in terms of cost. Of the estimated, the annual cost for dengue illness (SEs in parenthesis) in Malaysia is US $\$ 42.4( \pm 4.3)$ including per capita cost US $\$ 4.73$ for 1000 population size $(n=1000)$ with disability-adjusted life-years equivalent to $8324 .{ }^{20}$ As stated earlier, dengue-induced disease burden related to cost of care and mortality is of particular importance, especially in the low-income, middle-income countries where the dengue is endemic. ${ }^{15}$ Early identification of patients with dengue having high risk of prolonged hospital stay and death may serve as an effective tool to combat the upsurge of disease burden. In this attempt, we evaluated several factors associated with prolonged hospitalisation 
Table 3 Comparison of clinical characteristics (on-admission) of fatal and non-fatal dengue cases

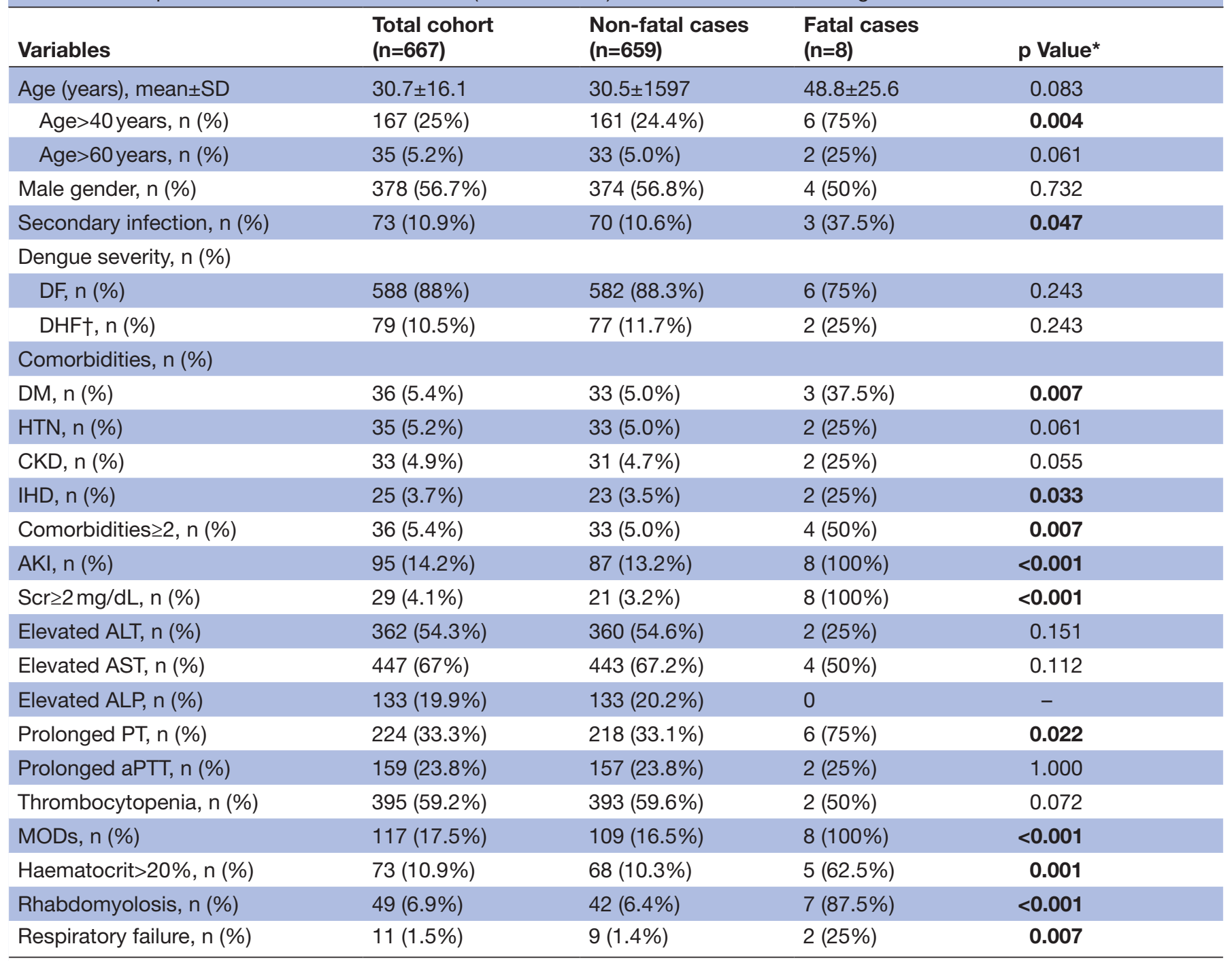

*p Values were calculated between patients with and without prolonged hospital stay.

†DHF refers to all grades including five cases of DSS.

AKI, acute kidney injury; ALP, alkaline phosphatase; ALT, alanine aminotransferase; aPTT, activated partial thromboplastin time; AST, aspartate aminotransferase; CKD, chronic kidney disease; DF, dengue fever; DHF, dengue haemorrhagic fever; DM, diabetes mellitus; DSS, dengue shock syndrome; HTN, hypertension; IHD, ischaemic heart disease; MODs, multiple organ dysfunction; PT, prothrombin time; Scr serum creatinine

and mortality among dengue patients attending tertiary care hospital.

Approximately half of the studied participants had prolonged hospitalisation that carry substantial economic, societal and personal burden. In our recent series, we reported that prolonged hospital stay is not only associated with DHF but also with classical DF. ${ }^{14}$ The proportion of prolonged hospitalisation in DF and DHF was $46.7 \%$ $(n=275 / 588)$ and $67.1 \%(n=53 / 79)$, respectively. These findings are in agreement with previous studies describing association of dengue infection with prolonged hospitalisation, irrespective of severity. ${ }^{14} 1521$ We found statistical association of DHF and prolonged hospital stay in the present study, where individuals with DHF had twice higher odds of longer hospital stay than patients without DHF. The mean hospital stay in the present series (4.47 days) is consistent with previous investigations reporting mean hospitalisation between 3.4 and 6.2 days. ${ }^{1421-24}$ Comparative analysis revealed that dengue patients with $\mathrm{HTN}$, elevated ALP, prolonged PT/aPTT and MODs were strongly associated $(\mathrm{p}<0.005)$ with hospital stay $>3$ days (table 1$)$. These findings are in concordance with the results of one and only study evaluating predictors of longer hospitalisation among patients with dengue. ${ }^{21}$

Many risk factors were identified and evaluated independently in their contribution to prolonged hospitalisation (table 2). Of these, presence of DHF, elevated ALP, prolonged PT/aPTT and MODs were found to be predisposing factors of longer hospital stay. Though Khalil et al reported old age and AKI as predictors of increased hospital stay, but the present study did not show any statistical association of these variables with LOS. ${ }^{21}$ Age was equally distributed among patients with 


\begin{tabular}{|c|c|c|c|c|c|c|c|c|c|}
\hline Sign and symptoms & P1 & P2 & P3 & P4 & P5 & P6 & P7 & P8 & n (\%) \\
\hline Fever & $x$ & $x$ & $x$ & $x$ & $x$ & $x$ & $x$ & $x$ & $8(100 \%)$ \\
\hline Nausea & $x$ & $x$ & $x$ & $x$ & $x$ & $x$ & $x$ & $x$ & $8(100 \%)$ \\
\hline Vomiting & $x$ & $x$ & $x$ & $x$ & $x$ & $x$ & $x$ & $x$ & $8(100 \%)$ \\
\hline Abdominal pain & $x$ & $x$ & $x$ & $x$ & $x$ & $x$ & $x$ & $x$ & $8(100 \%)$ \\
\hline Retro-orbital pain & $x$ & $x$ & $x$ & $x$ & & $x$ & $x$ & & $6(75 \%)$ \\
\hline Dysuria & $x$ & $x$ & & $x$ & $x$ & & $x$ & $x$ & $6(75 \%)$ \\
\hline Chills & $x$ & & $x$ & $x$ & & $x$ & & & $4(50 \%)$ \\
\hline Headache & & $x$ & $x$ & & & $x$ & $x$ & & $4(50 \%)$ \\
\hline Myalgia & & $x$ & & & & $x$ & $x$ & $x$ & $4(50 \%)$ \\
\hline Diarrhoea & $x$ & $x$ & & $x$ & & & $x$ & & $4(50 \%)$ \\
\hline Malaise & & $x$ & $x$ & & & $x$ & $x$ & & $4(50 \%)$ \\
\hline Lethargy & & $x$ & & & $x$ & & $x$ & $x$ & $4(50 \%)$ \\
\hline Restlessness & & & & & $x$ & & & $x$ & $2(25 \%)$ \\
\hline Jaundice & & & $x$ & & $x$ & $x$ & & $x$ & $4(50 \%)$ \\
\hline Rigours & & $x$ & $x$ & & & $x$ & $x$ & & $4(50 \%)$ \\
\hline Skin rash & $x$ & $x$ & & $x$ & & & $x$ & & $4(50 \%)$ \\
\hline Pleural effusion & $x$ & & & & & & $x$ & & $2(50 \%)$ \\
\hline Shortness of breath & $x$ & & & & & & $x$ & & $2(25 \%)$ \\
\hline Dizziness & & & & $x$ & & & & $x$ & $2(25 \%)$ \\
\hline Anorexia & & $x$ & & & & & $x$ & & $2(25 \%)$ \\
\hline Confusion & & $x$ & & & & & $x$ & & $2(25 \%)$ \\
\hline Asthenia & & & & & $x$ & & & $x$ & $2(25 \%)$ \\
\hline Petechia/purpura & $x$ & $x$ & & & & & & & $2(25 \%)$ \\
\hline Gum bleeding & $x$ & $x$ & & & & & & & $2(25 \%)$ \\
\hline Splenomegaly & & & & & $x$ & & $x$ & & $2(25 \%)$ \\
\hline Hepatomegaly & $x$ & & & & & & $x$ & & $2(25 \%)$ \\
\hline Conjunctivitis & & & $x$ & & & & $x$ & & $2(25 \%)$ \\
\hline Oedema & $x$ & & & $x$ & & & & & $2(25 \%)$ \\
\hline Ascites & $x$ & & $x$ & & & & & & $2(25 \%)$ \\
\hline Anasarca & $x$ & & & $x$ & & & & & $2(25 \%)$ \\
\hline
\end{tabular}

All the signs and symptoms listed were presented at hospital admission.

P1-P8 demonstrated patients numbers from 1 to 8 .

and without longer hospital stay in our study (table 1). It might be due to population differences, as Khalil and colleagues included patients aged $>14$ years while we included patients aged $\geq 12$ years in our study. Several previous case series have demonstrated the association of elevated SCr or AKI with prolonged hospitalisation and death. ${ }^{14} 15212526$ Although the prevalence of AKI in our study was high (14.2\%) but was equally distributed $(\mathrm{p}=0.242)$ among patients with and without prolonged hospitalisation (table 1). However, subgroup analysis showed that patients with AKI had significantly $(\mathrm{p}<0.001)$ longer duration of hospital stay than patients without AKI. Our findings, in addition to several other studies, suggest the monitoring of serum creatinine while managing patients with dengue.
Significant number of patients in our study had coagulopathy attributed to low platelets, deranged PT, aPTT and elevated liver transaminases (table 1). More than half of the study participants with prolonged PT and aPTT were associated with longer hospitalisation (table 1). Involvement of liver has been well documented during the course of dengue infection. ${ }^{27}$ However, only elevated ALP was observed as an independent predictor of longer hospital stay. Moreover, dysfunction of several other organs including brain, heart, muscles, spleen and gallbladder has recently emerged as expanded dengue syndrome and might contribute directly to the viral localisation in organs or to development of ischaemia of various organs as a result of AKI, coagulopathy and 
Table 5 Demographics, comorbidities, clinical features and causes of death of fatal dengue patients seen at HUSM between 2008 and 2013

\begin{tabular}{|c|c|c|c|c|c|c|c|}
\hline Patients & $\begin{array}{l}\text { Age (years) } \\
\text { /gender }\end{array}$ & $\begin{array}{l}\text { Duration of } \\
\text { illness prior } \\
\text { to admission } \\
\text { (day) }\end{array}$ & $\begin{array}{l}\text { Secondary } \\
\text { infection }\end{array}$ & Comorbidities & $\begin{array}{l}\text { Dengue } \\
\text { diagnosis }\end{array}$ & $\begin{array}{l}\text { Duration } \\
\text { of hospital } \\
\text { stay/ } \\
\text { duration of } \\
\text { fever }\end{array}$ & $\begin{array}{l}\text { Complications/ causes of } \\
\text { death }^{\star}\end{array}$ \\
\hline $\mathrm{P} 1$ & $14 / \mathrm{M}$ & 6 & Yes & Nil & DHF & $4 / 2$ & $\begin{array}{l}\text { DHF complicated with MODs, } \\
\text { severe AKI, rhabdomyolosis, } \\
\text { respiratory failure, bleeding } \\
\text { tendencies, ARDS }\end{array}$ \\
\hline $\mathrm{P} 2$ & $78 / \mathrm{F}$ & 7 & No & $\begin{array}{l}\text { IHD, HTN, } \\
\text { newly } \\
\text { diagnosed OP }\end{array}$ & DF & $6 / 4$ & $\begin{array}{l}\text { Septic shock, probable } \\
\text { leptospirosis, DF complicated } \\
\text { with MODs, severe AKI, } \\
\text { septicaemia, rhabdomyolosis, } \\
\text { bleeding tendencies, IHD, } \\
\text { HTN }\end{array}$ \\
\hline P4 & $13 / \mathrm{M}$ & 5 & No & Nil & DF & $3 / 2$ & $\begin{array}{l}\text { DF complicated with MODs, } \\
\text { severe AKI, dehydration } \\
\text { secondary to dengue fever, } \\
\text { rhabdomyolosis, respiratory } \\
\text { failure, ARDS }\end{array}$ \\
\hline P5 & $59 / \mathrm{M}$ & 6 & No & CKD, DM & DF & $3 / 2$ & $\begin{array}{l}\text { Acute on chronic renal failure, } \\
\text { severe AKI, DF complicated } \\
\text { with MODs, rhabdomyolosis, } \\
\text { DM, CKD }\end{array}$ \\
\hline P6 & $44 / F$ & 5 & No & Nil & DF & $12 / 2$ & $\begin{array}{l}\text { Severe AKI, DF complicated } \\
\text { with MODs, DIC, dehydration } \\
\text { secondary to dengue } \\
\text { fever, gastric disturbances, } \\
\text { hypotension }\end{array}$ \\
\hline P8 & $60 / \mathrm{M}$ & 5 & Yes & DM, CKD & DF & $3 / 2$ & $\begin{array}{l}\text { Renal complications, acute } \\
\text { on chronic renal failure, DF } \\
\text { complicated with MODs, } \\
\text { severe AKI, ketoacidosis, } \\
\text { rhabdomyolosis, DM, CKD }\end{array}$ \\
\hline
\end{tabular}

P1-P8 indicate number of patients from 1 to 8.

AKI, acute kidney injury; ARDS, acute respiratory distress syndrome; CKD, chronic kidney disease; DF, dengue fever; DHF, dengue haemorrhagic fever; DM, diabetes mellitus; F, female; HTN, hypertension; HUSM, Hospital University Sains Malaysia; IHD, ischaemic heart disease; M, male; MODs, multiple organ dysfunction.

old age. ${ }^{1628}$ Dengue patients with MODs had two times higher risk of longer hospital stay in the present study. Notably, DHF in combination with MODs, HTN, coagulopathy and elevated ALP might denote seriously sick patients who can potentially have more morbidity in the form of increased hospital stay. Identification of these patients at the earliest and their management with special care would be advantageous in reducing morbidity and hence their bed occupancy in the hospital.

DVIs are rarely fatal, although fatal infections do occur due to plasma leakage, fluid accumulation, respiratory distress, severe bleeding or MODs. ${ }^{14}$ Overall mortality in the current study was $1.1 \%$, which is consistent with previous national $^{1314}$ and global studies. ${ }^{21} 2629$ Malaysia 
experienced large dengue epidemics in the past decade affecting predominantly adults. ${ }^{5}$ This shift from childhood to adult illness is attributed to lower herd immunity and transmission outside home. ${ }^{514}$ Though the current study included patients aged $>12$ years but we did not observe any mortality among patients aged $<12$ years during the study period (data not shown). Two out of eight death cases belonged to the adolescent age group while the remaining had advanced age $>40$ years (tables 3 and 5). Interestingly, dengue patients aged $>40$ years were significantly associated with mortality in the current study. The higher risks of dengue-related deaths with increasing age might be contributed to decline in physiological functions and underline diseases in ageing people. In addition to comorbidities, older patients would have rehabilitation issues which may complicate admission and extend hospital stay. Dengue patients aged $>50$ years were reported to be at high risk of hospitalisation and mortality in two separate series. ${ }^{30}{ }^{31}$ Prolonged hospitalisation as defined in the current study was observed in $50 \%$ $(n=4 / 8)$ of fatal cases. This study explicitly illustrates that in addition to high mortality rate the advanced age is also associated with increased length of hospitalisation. These findings are consistent with a Taiwanese study, suggesting the impact of increasing age with higher fatality and longer hospitalisation. ${ }^{32}$ On the other hand, it can also be assumed that dengue patients with advanced age staying $>3$ days in the hospital should be considered as high-risk patients for mortality. However, we recommend large multicentric-controlled studies to evaluate this assumption. On the other hand, we found equal distribution of gender among fatal cases (table 3) and this is in contrast with the findings of a Malaysian study reporting preponderance of dengue deaths among females. ${ }^{13}$

It is interesting to note that all fatal cases had severe AKI and multiple MODs (tables 3 and 5). These findings are in agreement with the results of Khalil et al, who reported AKI in all fatal cases. ${ }^{21}$ In another case series, Lee $e t$ al reported the presence of AKI in $80 \%$ patients who died. ${ }^{32}$ Moreover, AKI as a cause of death has been documented in two separate studies reporting prevalence of AKI in $30 \%$ and $14 \%$ of fatal cases. ${ }^{1329}$ We also presented similar findings where severe AKI was among documented causes of death (table 5). This study also found that secondary infection, DM, IHD, prolonged PT, haematocrit $>20 \%$, rhabdomyolosis and respiratory failure were associated with dengue-related mortality in the overall study population (table 3). Current data also suggest that rhabdomyolosis and coagulation disorders are not uncommon intricacies in dengue infection, implicating that clinicians should be alert to the possible occurrence of these complications when taking care of patients with dengue.

Secondary infection usually occurs in dengue endemic regions and correlates to disease severity. ${ }^{15}$ Three of the fatal cases in this series had secondary infection while the remaining five cases had evidence of primary infection (table 5). Although this observation is in contrast to the other reports illustrating occurrence of mortality predominantly in secondary infection ${ }^{11} 1330$ but in agreement with the findings of Ong $e t a l$, where three out of seven death cases had primary infection. ${ }^{29}$

Mortality is usually linked to delayed provision of supportive treatment and/or premorbid chronic illness. ${ }^{1416}$ All death cases were admitted on day 5 (range 5-8 days) of illness and most of them had defervescence, followed by rapid deterioration of clinical condition. In our study, the average duration of illness prior to hospitalisation among death cases was significantly different from those who survived $(p=0.025)$. Our observation is consistent with earlier studies done on dengue deaths where late hospitalisation was found to be a possible contributing factor to increased risk of mortality. ${ }^{11} 1329$ These findings suggest that early care-seeking behaviour may determine the possibility of receiving and thereby avoiding fatal outcomes.

Besides care-seeking behaviour pattern, the presence of comorbidities is of paramount importance among patients with dengue. Several reports suggested that worsening of comorbid conditions, rather than directly from dengue infection, could be the reason for death seen especially in adults. ${ }^{33}$ Half of the death cases in the present study had underlying comorbid illness including CKD, DM, HTN and IHD. Patients with diabetes and IHD had higher risks of death compared with their survived counterparts (table 3). Several earlier reports implicated DM as a possible contributing factor to death. ${ }^{33}$ Nonetheless, $50 \%$ death cases were healthy individuals without any comorbid condition. This observation highlighted that underlying comorbidities might not be the main factor contributed to the dengue mortality in our cohort.

Good early predictors of mortality are presently lacking. Several reports emphasised the importance of individual warning signs as tool to recognise patients having high risk of severe illness and death. ${ }^{11}{ }^{34}{ }^{35}$ All the fatal cases in our study had at least one warning sign (table 4) and these findings are consistent with a Cuban study reporting presence of warning signs in all 12 fatalities. ${ }^{11}$ Contrary to these findings, Ong et al reported presence of warning signs in $50 \%$ of death cases. ${ }^{29}$ Hepatomegaly commonly occurs in severe dengue ${ }^{36}$ and was observed in two patients in our series. Hepatosplenomegaly usually associated with macrophage activation syndrome and was present in only one patient, suggesting its association with dengue infection. Such association has also been previously reported in the literature. ${ }^{37}$ These observations underscore that attention should be paid to uncommon presentations because they may often be related to poor prognosis and high mortality. The causes of death in the current study are consistent with those reported in other series on dengue fatal cases. ${ }^{11} 1338$

This retrospective study identified several factors associated with prolonged hospital stay and mortality among patients with dengue attending tertiary care hospital. These factors should be identified at the earliest and treated preferably in a special care set-up. Use of advanced 
therapies (renal replacement, ventilator support), along with inotropes or antibiotics (for haemodynamic disturbances and sepsis), can potentially improve patients' outcomes, which in turn can translate to reduced dengue-related hospital stay and mortality.

\section{STUDY LIMITATIONS}

However, several shortcomings of the present study should be considered. This is a single-centre study with retrospective analysis and the results may therefore not necessarily be generalised to the other populations. Additionally, this study depends on thoroughness of clinicians' documentation, so disease severity and clinical outcomes of included patients may be biased due to lack of standardised management protocol for dengue. The admission and discharge criteria may vary among various clinicians attending patients and may interfere with the results of our findings. The small number of fatal cases in our study may make statistical power quite small for identification of factors associated with dengue-related mortality. However, four case series with limited number of fatal cases $(n=7,5,19,18)$ evaluating factors of mortality support the findings of present study. ${ }^{29} 323839$ A case-control study with much larger sample size is needed to determine whether clinico-laboratory findings seen in our series are exclusive to fatal cases of dengue or seen equally in non-fatal cases. Moreover, the study population included adults and hence results cannot be generalised to paediatric patients. Unfortunately, autopsy and postmortem data of dead patients were not available. Last but not least, WHO 1997 criteria of dengue classification were used in the current study because similar criteria are being used in Malaysia. A recent investigation has also verified diagnosis of dengue using WHO 1997 classification in Malaysia. ${ }^{40}$ However, health authorities included WHO 2009 criteria in new guidelines on dengue infection that issued in November 2016.

Nevertheless, the strength of this study lies in our attempt to include a large heterogeneous cohort of dengue population. The present study improves awareness of factors associated with prolonged hospitalisation and death among patients with dengue. It also highlights the need for more studies and for strategised management protocol in order to reduce the disease burden. Moreover, this is the only largest study conducted in Malaysia describing factors relating to prolonged hospitalisation. These observations, along with our previous case series, ${ }^{1415182528}$ prove to be a valuable addition in dengue literature and warrant further investigations.

\section{CONCLUSION}

Our case series demonstrates high proportion of dengue patients with prolonged hospital stay. Patients with severe disease (DHF) along with elevated ALP levels and deranged PT/aPTT had higher likelihood to stay $>3$ days in the hospital. Our observations exemplified that fatal dengue infection does occur in adults and in primary infection, irrespective of gender. Factors including advance age, secondary infection, comorbidities, prolonged PT, haemoconcentration and rhabdomyolosis were found to be statistically associated with mortality. Early identification of dengue patients with these factors will have obvious advantages in terms of appropriate decisions about treatment and management in high dependency units. Attention to these high-risk patients may have a positive impact on both patients and healthcare system by reducing dengue-related bed occupancy and mortality.

\section{RECOMMENDATION}

For clinical practice, doctors treating dengue, especially those working in the front line, should be made aware of the factors significantly associated with longer hospital stay and dengue mortality. With proper identification of these factors, time and resources can be focused on those at highest risk.

Correction notice This article has been corrected since it was first published. Amer Hayat Khan has been mentioned as the second Corresponding author.

Contributors THM, YHK and ASA provided substantial contributions to the conception or design of the work. THM and AS were involved in the acquisition, analysis or interpretation of data. THM and AHK were responsible for drafting the work or revising it critically for important intellectual content. AHK and ASA gave final approval of the version to be published. All the authors substantially contributed to the manuscript and are accountable for all aspects of the work in ensuring that questions related to the accuracy or integrity of any part of the work are appropriately investigated and resolved.

Competing interests None declared.

Ethics approval The study was approved by Human Resource Ethics Committee (JEPeM) of HUSM (USM/JEPeM/14080278).

Provenance and peer review Not commissioned; externally peer reviewed.

Data sharing statement All the data relating to this study are available within manuscript.

Open Access This is an Open Access article distributed in accordance with the Creative Commons Attribution Non Commercial (CC BY-NC 4.0) license, which permits others to distribute, remix, adapt, build upon this work non-commercially, and license their derivative works on different terms, provided the original work is properly cited and the use is non-commercial. See: http://creativecommons.org/ licenses/by-nc/4.0/

(C) Article author(s) (or their employer(s) unless otherwise stated in the text of the article) 2017. All rights reserved. No commercial use is permitted unless otherwise expressly granted.

\section{REFERENCES}

1. World Health Organization. Dengue and severe dengue. Fact sheet No117 (UpdatedApril 2016). http://www. who. int/mediacentre/ factsheets/fs117/en (accessed: Mar 2016).

2. Deen JL, Harris E, Wills B, et al. The WHO dengue classification and case definitions: time for a reassessment. Lancet 2006;368:170-3.

3. http://www.bebasdenggi.my/dengue-hotspot-area.php

4. Monath TP. Dengue: the risk to developed and developing countries. Proc Natl Acad Sci U S A 1994;91:2395-400.

5. Cheah WK, Ng KS, Marzilawati AR, et al. A review of dengue research in malaysia. Med J Malaysia 2014;69:59-67.

6. World Health Organization. Country Co-operation Strategy. Malaysia at Glance. http://www.who.int/countryfocus/cooperation_strategy/ ccsbrief_mys_en.pdf (accessed Mar 2016).

7. http://www.bbc.com/news/world-asia-29320119

8. http://outbreaknewstoday.com/malaysia-dengue-outbreak-nears100000 
9. http://outbreaknewstoday.com/malaysia-dengue-death-toll-up-to126

10. WPRO dengue situation update 481. http://www.wpro.who.int/ emerging diseases/dengue_biweekly_20151229.pdf?ua=1

11. Guzmán MG, Alvarez M, Rodríguez R, et al. Fatal dengue hemorrhagic fever in Cuba, 1997. Int J Infect Dis 1999;3:130-5.

12. Ooi EE, Goh KT, Chee Wang DN. Effect of increasing age on the trend of dengue and dengue hemorrhagic fever in Singapore. Int $J$ Infect Dis 2003;7:231-2.

13. Sam SS, Omar SF, Teoh BT, et al. Review of dengue hemorrhagic fever fatal cases seen among adults: a retrospective study. PLOS Negl Trop Dis 2013;7:e2194.

14. Mallhi TH, Khan AH, Adnan AS, et al. Clinico-laboratory spectrum of dengue viral infection and risk factors associated with dengue hemorrhagic fever: a retrospective study. BMC Infect Dis 2015;15:1.

15. Mallhi TH, Khan AH, Adnan AS, et al. Incidence, characteristics and risk factors of acute kidney injury among dengue patients: a retrospective analysis. PLoS One 2015;10:e0138465.

16. World Health Organization. Comprehensive guidelines for prevention and control of dengue and dengue hemorrhagic fever. 2011.

17. Khan NA, Azhar El, El-Fiky S, et al. Clinical profile and outcome of hospitalized patients during first outbreak of dengue in Makkah, Saudi Arabia. Acta Trop 2008;105:39-44.

18. Mallhi TH, Khan AH, Sarriff A, et al. Defining acute kidney injury in dengue viral infection by conventional and novel classification systems (AKIN and RIFLE): a comparative analysis. Postgrad Med J 2016;92:78-86.

19. Bursac Z, Gauss CH, Williams DK, et al. Purposeful selection of variables in logistic regression. Source Code Biol Med 2008;3:17.

20. Suaya JA, Shepard DS, Siqueira JB, et al. Cost of dengue cases in eight countries in the Americas and Asia: a prospective study. Am J Trop Med Hyg 2009;80:846-55.

21. Khalil MA, Tan J, Khalil MA, et al. Predictors of hospital stay and mortality in dengue virus infection-experience from Aga Khan University Hospital Pakistan. BMC Res Notes 2014;7:473.

22. Goh KT, Ng SK, Chan YC, et al. Epidemiological aspects of an outbreak of dengue fever/dengue haemorrhagic fever in Singapore. Southeast Asian J Trop Med Public Health 1987;18:295-302.

23. Lye DC, Chan M, Lee VJ, et al. Do young adults with uncomplicated dengue fever need hospitalisation? A retrospective analysis of clinical and laboratory features. Singapore Med J 2008;49:476.

24. Agarwal R, Kapoor S, Nagar R, et al. A clinical study of the patients with dengue hemorrhagic fever during the epidemic of 1996 at Lucknow, India. Southeast Asian J Trop Med Public Health 1999;30:735-40.
25. Mallhi TH, Khan AH, Sarriff A, et al. Association of ward acquired, on-admission, progressive and non-progressive AKI with death among dengue patients: a hidden relationship. Acta Med Port 2016;29:157-8

26. Khalil MA, Sarwar S, Chaudry MA, et al. Acute kidney injury in dengue virus infection. Clin Kidney J 2012;5:390-4.

27. Seneviratne SL, Malavige GN, de Silva HJ. Pathogenesis of liver involvement during dengue viral infections. Trans $R$ Soc Trop Med Hyg 2006;100:608-14.

28. Mallhi TH, Sarriff A, Adnan AS, et al. Dengue-induced Acute Kidney Injury (DAKI): a neglected and fatal complication of dengue viral infection-a systematic review. J Coll Physicians Surg Pak 2015;25:828-34.

29. Ong A, Sandar M, Chen Ml, et al. Fatal dengue hemorrhagic fever in adults during a dengue epidemic in Singapore. Int $J$ Infect Dis 2007;11:263-7.

30. Guzmán MG, Kouri G, Bravo J, et al. Effect of age on outcome of secondary dengue 2 infections. Int $J$ Infect Dis 2002;6:118-24.

31. García-Rivera EJ, Rigau-Pérez JG. Dengue severity in the elderly in Puerto Rico. Rev Panam Salud Publica 2003;13:362-8.

32. Lee IK, Liu JW, Yang KD. Clinical and laboratory characteristics and risk factors for fatality in elderly patients with dengue hemorrhagic fever. Am J Trop Med Hyg 2008;79:149-53

33. Liu C-C, Huang K-J, Huang M-C, et al. High case-fatality rate of adults with dengue hemorrhagic fever during an outbreak in nonendemic Taiwan: risk factors for dengue-infected elders. Am J Infect Dis 2008;4:10-17.

34. Rigau-Pérez JG, Laufer MK. Dengue-related deaths in Puerto Rico, 1992-1996: diagnosis and clinical alarm signals. Clin Infect Dis 2006;42:1241-6.

35. Chan KP, Lau GK, Doraisingham S, et al. Adult dengue deaths in Singapore. Clin Diagn Virol 1995;4:213-22.

36. Thein TL, Gan VC, Lye DC, et al. Utilities and limitations of the World Health Organization 2009 warning signs for adult dengue severity. PLoS Negl Trop Dis 2013;7:e2023.

37. Ray S, Kundu S, Saha M, et al. Hemophagocytic syndrome in classic dengue fever. J Glob Infect Dis 2011;3:399.

38. Almas A, Parkash O, Akhter J. Clinical factors associated with mortality in dengue infection at a tertiary care center. Southeast Asian J Trop Med Public Health 2010;41:333-40.

39. Branco MR, Luna EJ, Braga Júnior LL, et al. Risk factors associated with death in Brazilian children with severe dengue: a case-control study. Clinics 2014;69:55-60.

40. Liew SM, Khoo EM, Ho BK, et al. Dengue in Malaysia: factors associated with dengue mortality from a National Registry. PLoS One 2016;11:e0157631 